\title{
Metataxonomic and Histopathological Study of Rabbit Epizootic Enteropathy in Mexico
}

\author{
Xiao-Haitzi Daniel Puón-Peláez ${ }^{1}$, Neil Ross McEwan ${ }^{2}{ }^{\mathbb{D}}$, José Guadalupe Gómez-Soto ${ }^{3}{ }^{(D)}$, \\ Roberto Carlos Álvarez-Martínez ${ }^{4}$ and Andrea Margarita Olvera-Ramírez ${ }^{5, * \mathbb{D}}$
}

1 Doctorado en Ciencias Biológicas, Facultad de Ciencias Naturales, Universidad Autónoma de Querétaro, Avenida de las Ciencias S/N Juriquilla, Delegación Santa Rosa Jáuregui, Santiago de Querétaro, Qro. C.P. 76230, Mexico; xh.puon@gmail.com

2 School of Pharmacy \& Life Sciences, Robert Gordon University, Garthdee Road, Aberdeen AB10 7GJ, UK; n.mcewan@rgu.ac.uk

3 Cuerpo Académico de Nutrición y Reproducción Animal, Facultad de Ciencias Naturales, Universidad Autónoma de Querétaro, Avenida de las Ciencias S/N Juriquilla, Delegación Santa Rosa Jáuregui, Santiago de Querétaro, Qro. C.P. 76230, Mexico; jose.gomez@uaq.mx

4 Licenciatura en Microbiología, Facultad de Ciencias Naturales, Universidad Autónoma de Querétaro, Av. Junipero Serra, Antiguo Aeropuerto, Campus Aeropuerto S/N. Santiago de Querétaro, Qro. C.P. 76140, Mexico; roberto.alvarez@uaq.mx

5 Cuerpo Académico Salud Animal y Microbiología Ambiental, Facultad de Ciencias Naturales, Universidad Autónoma de Querétaro, Avenida de las Ciencias S/N Juriquilla, Delegación Santa Rosa Jáuregui, Santiago de Querétaro C.P. 76230, Mexico

* Correspondence: andrea.olvera@uaq.mx; Tel.: +52-442-192-1200 (ext. 5316)

Received: 21 April 2020; Accepted: 24 May 2020; Published: 28 May 2020

Simple Summary: Epizootic rabbit enteropathy (ERE) is a worldwide-distributed dysbiotic syndrome that affects young rabbits. In Mexico, ERE represents 32\% of the enteropathies that occur in rabbit production farms. The etiology of this syndrome has not been clarified yet; however, it has been associated with nutritional, environmental, and microbial factors. A metataxonomic and histopathology study of ERE was carried out to compare the lesions and gastrointestinal microbiota of healthy and positive-ERE rabbits. The results revealed a difference in the diversity and abundance of the gastrointestinal microbiota in rabbits with ERE. The genus Clostridium and the species. Cloacibacillus porcorum and Akkermansia muciniphila were associated with the presentation of ERE. Histopathologic analysis showed smaller crypt sizes in the colon of ERE rabbits.

Abstract: Epizootic rabbit enteropathy (ERE) affects young rabbits and represents $32 \%$ of the enteropathies in rabbit production farms in Mexico. The etiology of this syndrome has not been clarified yet. A metataxonomic and histopathology study of ERE was carried out to compare the gastrointestinal microbiota and histopathological lesions of healthy and positive-ERE rabbits. The metataxonomic study was done using an Illumina MiSeq (MiSeq ${ }^{\circledR}$ system, Illumina, San Diego California, USA) massive segmentation platform, and a Divisive Amplicon Denoising Algorithm 2 (DADA2 algorithm) was used to obtain Shannon and Simpson diversity indices as well as the relative abundance of the identified communities. For the histopathological study, paraffin sections of the cecum, ileo-cecal valve, and colon were stained with eosin and hematoxylin. AxioVision 4.9 software (Carl Zeiss MicroImaging GmbH, Jena, Germany) was used to measure the crypt depths. Statistical analysis was done using PERMANOVA analysis for the metataxonomic study and ANOVA for the histopathology study. Histopathologic analysis showed smaller sizes of crypts in the colon of ERE rabbits. Differences were observed in the diversity and abundance of the gastrointestinal microbiota between the analyzed groups. The genus Clostridium and the species Cloacibacillus porcorum and Akkermansia muciniphila were associated with ERE. The results obtained from this study can provide information for future clarification of the etiology and proposals of effective treatments. 
Keywords: rabbits; ERE; etiology

\section{Introduction}

Rabbit epizootic enteropathy (ERE) is a digestive syndrome, which has had a negative impact on rabbit production since the 1990s [1,2]. ERE can affect up to $95 \%$ of animals in any production system and it can reach levels of approximately $90 \%$ morbidity and $80 \%$ mortality [3]. Rabbits between 3 and 7 weeks are highly susceptible to ERE and reduce their daily food consumption for approximately 2 weeks, which causes a decrease in growth and daily weight gain [3,4].

In Mexico, ERE was first observed towards the end of 2001 or the beginning of 2002, affecting different production centers, but mainly in rabbits between 5 and 7 weeks of age [5]. An incidence of abundance of $32 \%$ has been reported with ERE [6], presenting highly variable mortality rates ranging from $30 \%$ to $70 \%$ [7]. Non-specialized feeding in semi-intensive systems in rabbit production increases the risk of developing ERE in Mexico. Additionally, the microbial profile of the digestive tract of the female rabbit determines the profile of her litter [8], meaning, if the mother has a dysbiosis in her microbial profile, the entire litter will be affected.

ERE can be difficult to diagnose due to the similarity of the signs between this and other enteropathies [9]. During ERE outbreaks, rabbits reduce food and water intake, and in extreme cases, they will stop eating and drinking. Affected rabbits can show mild diarrhea and translucent mucus $[7,10]$. However, the most common signs are abdomen bloating, generalized dilatation in the gastrointestinal tract, cecal paralysis in some cases, and the presence of abundant mucus [11].

After necropsy, animals can show a distended stomach and small intestine with the presence of gaseous and aqueous contents. In the cecum, there is translucent and prominent mucus [10,12,13]. ERE is characterized by an absence of any inflammatory or congestive lesions; however, goblet cell hyperplasia has been described in the small intestine $[4,9,14]$. In addition, certain chemical alterations lead to changes in the $\mathrm{pH}$ of the ileum and colon [10]. There is a decrease in the $\mathrm{pH}$ of the stomach, as well as in part of the duodenum and urine. This decrease in $\mathrm{pH}$ is believed to be due to the lack of food in the stomach, while the increase in $\mathrm{pH}$ in the colon is due to microbial dysbiosis $[4,15]$.

The first metataxonomic studies on ERE reported changes in the abundances of certain microorganisms, such as: Clostridium spp., Bacteroides spp., Ruminococcus spp. [16], Akkermansia muciniphila, Bacteroides-Prevotella, Clostridium coccoides, Methanobrevi bacter [15], Bacteroides, Blautia, Dorea, Clostridia, and a number of unclassified organisms [17]. These studies used the second-generation platform 454 pyrosequencing. Recently, Jin et al. [18] used a second-generation platform Illumina, MiSeq system. They reported a change in six genera (Bacteroides, Rikenella, Akkermansia, Escherichia, Lysinibacillus, and Clostridium), meaning organisms from five families (Bacteroidaceae, Verrucomicrobiaceae, Enterobacteriaceae, Planococcaceae, and Clostridiaceae) and three phyla (Bacteroidetes, Verrucomicrobia, and Firmicutes) were affected. They also reported the presence of the phylum Sinergetes, mainly the genus Cloacibacillus porcorum, which is related to the gastrointestinal microbiome in pigs [19] and oral problems (periodontitis) in bovines [20].

In the present work, a histopathological study and metataxonomic analysis was performed using a second-generation Illumina MiSeq sequencing [21] of the V3-V4 region of the ribosomal 16s gene to get the microbial profile of healthy and ERE rabbits of an intensive rabbit farm in Mexico.

\section{Materials and Methods}

The study was approved by the Bioethics Committee of the Faculty of Natural Sciences of the Autonomous University of Querétaro (number 93FCN2016).

The study was undertaken in a commercial meat rabbit production farm localized $19^{\circ} 45^{\prime}$ north latitude and $101^{\circ} 03^{\prime}$ west longitude, at an altitude of $1900 \mathrm{~m}$ above sea level (Jaripeo, Charo municipality, Morelia Michoacán, Mexico). This farm typically has 100 breeding animals ( 80 females and 20 males) 
and animals for meat production are housed at a typical density of 7 animals per $0.54 \mathrm{~m}^{2}$. Typically, in total, there are normally 660 animals housed in a single room (100 breeders and 560 for meat). Animals were fed with a standard commercial diet (crude protein: $16 \%$, crude fiber: $17 \%$ ) and had access to water ad libitum. The diet contained no anticoccodic or antibiotic compounds. All rabbits were weaned at 35 days old. Six rabbits aged 42 days with ERE (mean weight: $449.16 \mathrm{~g}$ ) were chosen; ERE diagnosis was determined based on clinical signs, such as diarrhea, distended abdomen, mucus excretion, cecal impaction, decrease of food intake, and bruxism [7,11]. In addition, six healthy rabbits (mean weight of $483.5 \mathrm{~g}$ ) were chosen from the same feeding and handling group. Selected rabbits were placed in individual cages $(60 \times 90 \times 40 \mathrm{~cm})$ in 2 groups: healthy and ERE positive. The groups were housed in the quarantine zone of the same farm in Morelia.

Euthanasia was performed during the same day for all animals according to NOM-062ZOO-1999 [22], by cervical dislocation through mechanical traction and jugular exsanguination. The healthy group was euthanized and sampled first in the trail area of the farm. Subsequently, the facilities and materials were cleaned and disinfected. The ERE group was then euthanized using the same protocol.

Immediately after being euthanized, an incision was made into the abdomen to expose the stomach, small intestine, cecum, and large intestine. A hemp thread ligation was performed in the final portion of the small intestine and another at the end of the ileo-ceco-colic valve. Subsequently, 2 ligatures were made for the cut, one $0.5 \mathrm{~cm}$ before the ligation of the small intestine and another $0.5 \mathrm{~cm}$ after the ligation of the ileo-ceco-colic valve, and the cut was performed between both ligatures.

To obtain intestinal tissue, a ligation was performed at the beginning of the ileo-ceco-colic valve, another at $10 \mathrm{~cm}$ after the ligation of the small intestine (beginning of the colon), and a further $3 \mathrm{~cm}$ from the previous ligation. Cuts were made to obtain the area in the ligated ileo-ceco-colic valve and colon, which were cleaned with phosphate buffered solution (PBS) and then infiltrated with $10 \%$ PBS buffered formalin ( $\mathrm{pH} 7$ ) and immersed in flasks with the same formalin composition.

In the case of the cecum, a longitudinal cut was made and washed with PBS, then it was held in place with pins on cork circles of $2 \mathrm{~cm}$ diameter and immersed in bottles with buffered $10 \%$ formalin ( $\mathrm{pH}$ 7). In this way, samples from the cecum, ileum, and colon were obtained for histopathology analysis. The bottles were stored at room temperature and transferred to the Animal Nutrition Laboratory, Natural Sciences Faculty of the Autonomous University of Queretaro (FCN, UAQ) for further analysis. Furthermore, the entire contents of the cecum were poured into a 15-mL RNase and DNase free plastic tube, followed by releasing the ligation of the small intestine. Once the tube was filled, this was immediately frozen in a $-20{ }^{\circ} \mathrm{C}$ freezer for storage and transport to the Laboratory Veterinary Microbiology, FCN, UAQ, where samples were stored at $-80^{\circ} \mathrm{C}$ for further analysis.

For histopathological analysis, the tissue samples were washed with saline and embedded in paraffin, then $5-\mu \mathrm{m}$ sections were cut from each sample. Staining was carried out using the hematoxylin and eosin technique. Stained slides were analyzed by light microscopy (Vert. A1, CarlZeiss Microscopy, Gottingen, Germany). Crypt depth was measured in the crypts when there was a complete longitudinal section, a villus, and its associated crypt. The depth of the crypt was measured from the crypt-villus junction to the base using AxioVision 4.9 software (Carl Zeiss MicroImaging GmbH, Jena, Germany). In total, 10 crypts were measured for each sample [23]. A completely randomized design and a Tukey test [24] with SAS 2008 software (Statistical Analysis System, SAS Institute, Cary, North Carolina, USA) were used for comparison between samples.

For metataxonomic analysis, the cecal content of each sample was freeze-dried as follows: $1.5 \mathrm{~mL}$ of cecal content of each sample was placed in a 2-mL tube free of DNases and RNAses. Tubes were covered with Parafilm and some holes were made in the Parafilm with a sterile needle. The tube was placed into a flask (Fast-Freeze ${ }^{\mathrm{TM}}$ Flasks, Labconco ${ }^{\mathrm{TM}}$, Kansas MO, USA). The flask was chilled for $24 \mathrm{~h}$ at $0{ }^{\circ} \mathrm{C}$. The refrigerated flask was placed in the freeze dryer (FreeZone ${ }^{\mathrm{TM}}$ Freeze-Dry Systems $4.5 \mathrm{~L}$, Labconco ${ }^{\mathrm{TM}}$, Kansas MO, USA) for $48 \mathrm{~h}$ of drying. 
DNA extraction of lyophilized cecal samples was performed with a QIAamp DNA Stool Mini Kit (Qiagen, Venlo, the Netherlands) following the manufacturer's instructions as follows. Disruption of cells was performed using $200 \mathrm{mg}$ of silica beads $(0.1 \mathrm{~mm})$ placed in a 2-mL tube free of DNases and RNAses. Afterwards, $50 \mathrm{mg}$ of lyophilized cecal sample and $1.4 \mathrm{~mL}$ of stool lysis buffer (ASL) were added. Homogenization with a vortex was performed for $1 \mathrm{~min}$ and the sample was heated for $5 \mathrm{~min}$ at $95^{\circ} \mathrm{C}$, homogenized by vortexing for $15 \mathrm{~s}$, and centrifuged for $1 \mathrm{~min}$ at $14,000 \times \mathrm{g}$. Then, $1.2 \mathrm{~mL}$ of sample was transferred to a new 2-mL tube and 1 InhibitEX tablet was added. Homogenization was performed for $1 \mathrm{~min}$ and incubated at room temperature for $1 \mathrm{~min}$. Centrifugation was performed for $5 \mathrm{~min}$ at $14,000 \times \mathrm{g}$. The supernatant was placed in a new $1.5-\mathrm{mL}$ tube along with $15 \mu \mathrm{L}$ of proteinase $\mathrm{K}$, $200 \mu \mathrm{L}$ supernatant, and $200 \mu \mathrm{L}$ of Lysis Buffer (AL) solution, which was mixed for $15 \mathrm{~s}$. This was incubated for $10 \mathrm{~min}$ at $70{ }^{\circ} \mathrm{C}$. After this, purification and elution were performed as described in the manufacturer's instructions, and DNA was eluted was stored at $-20{ }^{\circ} \mathrm{C}$.

A standardization of the DNA concentration of each sample was performed to give a concentration of $50 \mathrm{ng} / \mu \mathrm{L}$, and sequencing of the DNA of the cecal contents was performed with the second-generation DNA sequencing platform (MiSeq ${ }^{\circledR}$ system, Illumina, San Diego California, USA), where the V3-V4 region of the 16S rRNA gene was used (Zymo Research Corp Irvine California USA). Sequencing was performed using genomic DNA, i.e., without a prior round of polymerase chain reaction (PCR) before sequencing. The MiSeq ${ }^{\mathrm{TM}}$ sequencing used a v3 reagent kit with 600 cycles and $>10 \%$ PhiX spike-in and $2 \times 250$ bp chemistry.

The protocol after sequencing was as follows: Filtering, identifying unique sequences (Dereplication), constructing tables of operational taxonomic units, removing chimeric sequences, assigning taxonomic identification, and determining abundance and diversity.

Sequences were processed by filtering the low-quality reads and trimming the sequencing adapters, which was determined with the quality score function, and trim and filter function of the DADA2 package, which also removed chimeric sequences. This cut-off was used to achieve a maximum of 2 errors per read and a size of 540 in reads forward and 150 in reads reverse, leaving approximately $480 \mathrm{bp}$ readings with Trimmomatic software Bolger [25], and an inspection of the data quality was made with FastQC (Babraham Bioinformatics, Babraham, Cambridge, UK), and again later using the $\mathrm{R}$ Project software for Statistical Computing following the work guide proposed by Callahan et al. [21]. The following packages were used: Divisive Amplicon Denoising Algorithm 2 (DADA2), DECIPHER (DECIPHER Project Manage, Cambrige, UK), Phyloseq Microbiome, Vegan, and Ggplot2 (R packages, Wellignton New Zealand). Taxonomy assignment was performed using Uclust from Qiime v.1.9.1 (Quantitative Insights Into Microbial Ecology, Colorado, USA) and compared against the internally designed and curated Zymo 16S Research Database (Zymo Research California, USA). Composition visualization, alpha-diversity, and beta-diversity analyses were performed with Qiime v.1.9.1. For the comparison of the sequences identified by DADA2, a PERMANOVA test was performed following the methodology described in the Vegan package manual [26]. A rarefaction curve was produced with the rarefy script: Rarefaction Species Richness in Vegan: Community Ecology Package [26]. In the case of the analysis of bacterial diversity, this was evaluated by calculating the Shannon index, the Simpson index, the Chao1 index and an estimate of coverage based on abundance (ACE), following the methodology proposed in the Vegan package manual [26].

The operational taxonomic units (OTUs) and abundance tables were used for the linear discriminant analysis effect size (LEfSe) calculation. The metadata information compared (ERE vs. healthy) was used. Subsequently, the biomarkers of each class and visualization of the representative OTUs were generated.

\section{Results}

\subsection{Histopathological Study}

The findings at necropsy were consistent in all infected rabbits with ERE. The stomach was full of liquid, without the presence of food, and slightly ischemic. They also presented with an accumulation 
of liquid, mucus, and gas in the small intestine and colon, causing bowel distension and loss of visibility of the intestinal austras. The cecum had an accumulation of liquid and gas, as well as food; in two cases the food was impacted, and the liquid was scarce. In the case of healthy rabbits, no pathological change was observed (Figure 1A,B).

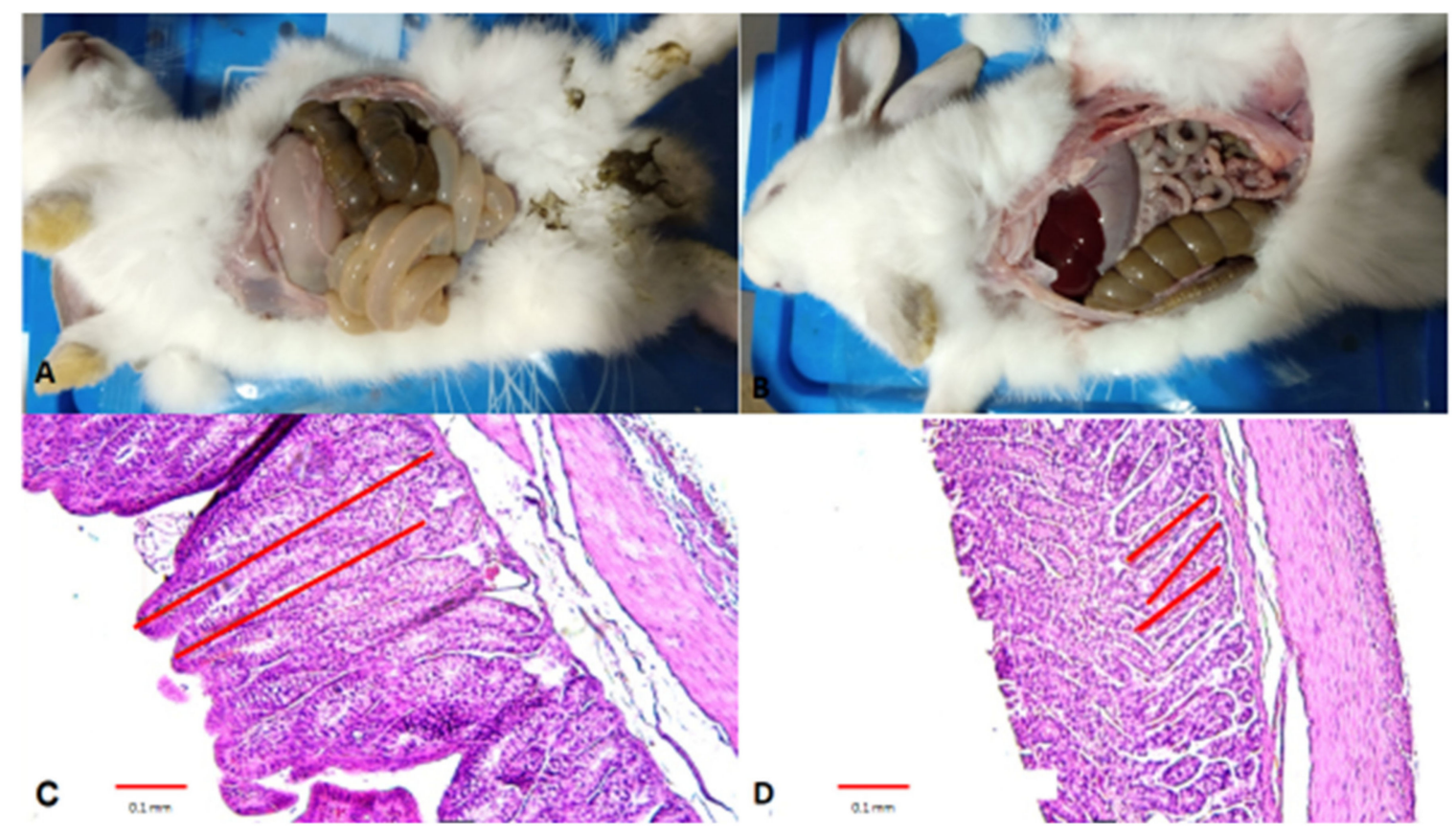

Figure 1. Histopathological study of rabbit digestive tracts. (A) Image of necropsy of a healthy rabbit; (B) Image of necropsy of an Epizootic rabbit enteropathy (ERE)-infected rabbit; (C) Section of villi of the colon of a healthy rabbit; (D) Section of villi from an ERE-infected rabbit colon. For (C,D), the red lines show the length of the crypts.

The histological sections of ERE rabbits were a smaller size of the crypts in the colon section (Figure 1C,D). In healthy sections, no change was reported in the crypts (Table 1). Other histological changes were not observed in the epithelium.

Table 1. Mean crypt depths in three regions of the rabbit intestine. Values shown are the mean of 10 crypts from the 6 animals per treatment; ERE+ (infected) versus ERE- (healthy).

\begin{tabular}{ccccc}
\hline \multirow{2}{*}{ Site } & \multicolumn{4}{c}{ Depth of Crypts $(\mu \mathrm{m})$} \\
\cline { 2 - 5 } & ERE+ & ERE- & SEM & $p$ Value \\
\hline Ileo-ceco-colic valve & 199 & 187 & 16.21 & 0.73 \\
Cecum & 136 & 121 & 5.55 & 0.22 \\
Colon & 173 & 308 & 10.51 & 0.0004 \\
\hline
\end{tabular}

\subsection{Metataxonomic Study}

\subsubsection{Bacterial Abundance}

The samples from the ERE-positive rabbits yielded more sequences relative to those from healthy animals (Figure 2). Following rarefaction curve analysis, an almost horizontal asymptotic curve was obtained for almost all individuals (Figure 3). This indicates that the number of species (based on the number of OTUs) was no longer increasing in relation to the number of sequenced amplicons included in the species identification. In addition, at the level of individual animals, there were more OTUs per rabbit in healthy animals than there were in those that had previously had the disease. 
Full details of the respective abundance of each Phylum, Class, Order, Family and Genus are shown in the Supplementary Table S1 In the ERE-positive rabbits, the most abundant phyla were: Firmicutes, Bacteroides, and Verrucomicrobia. The least abundant phyla detected were Tenericutes, Cyanobacteria, and Euryachaeota. In healthy rabbits, the most abundant phyla were Firmicutes, Verrucomicrobia, and Bacteroides, with Saccharibacteria, Cyanobacteria, and Tenericutes as the least abundant detectable phyla. The phylum Synergistetes was only seen in rabbits with ERE. In addition, there were a number of unidentified sequences (mean of 3505 for the group positive for ERE and mean of 2366 for the group without disease) reported (Table 2). In terms of the relative abundance, in rabbits with ERE, there was obviously more Verrucomicrobia and Bacteroides, whilst in healthy animals, there was obviously more Firmicutes (Figure 4).

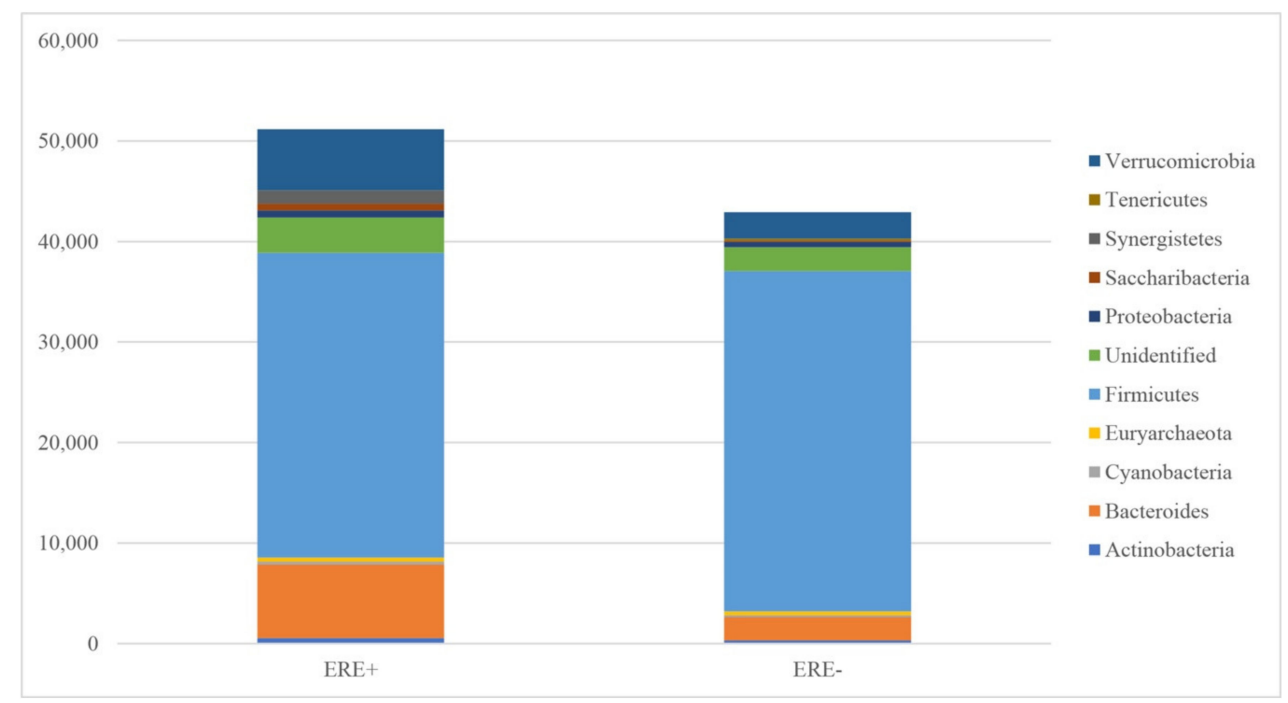

Figure 2. The abundance of bacterial genera in cecal samples of healthy and ERE rabbits. ERE+: Rabbits positive for epizootic enteropathy. ERE-: Healthy rabbits.

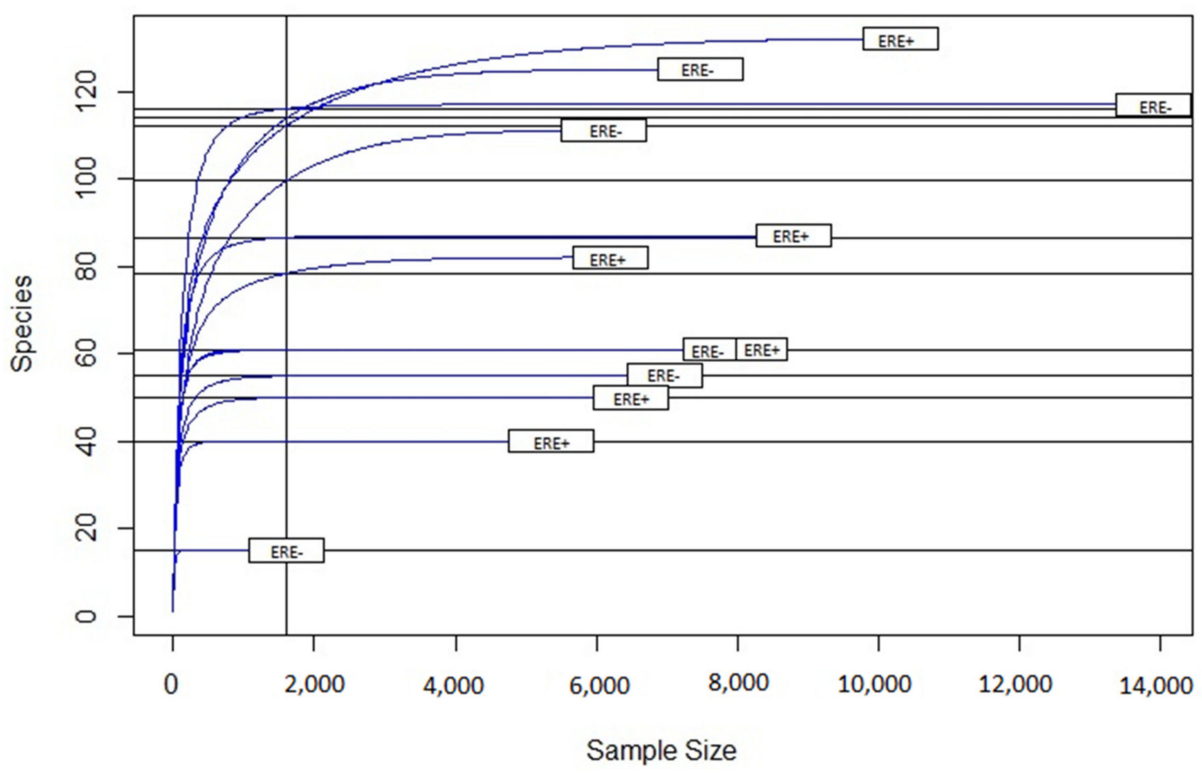

Figure 3. Rarefaction curve for sequence analysis. The vertical axis shows the number of different species (Operational taxonomic units, OTUs) that would be expected to be found after sampling the number of sequences shown on the horizontal axis. The curvature towards the horizontal indicates the greater sequencing effort required to observe new species. The curve was obtained with the Rarefaction Species Richness script from the Vegan package on the Rstudio platform [27]. 
Table 2. Mean abundance ( $n=6$ relative to health status) with standard error and percentage of the bacterial phyla of the cecal contents of rabbits with and without ERE. Where a phylum was not observed is denoted by "-".

\begin{tabular}{cccccc}
\hline Phylum & ERE+ & $\%$ & ERE- & $\%$ & $p$ Value \\
\hline Actinobacteria & $538 \pm 108.5$ & 1.05 & $312 \pm 217.9$ & 0.73 & 0.138 \\
Bacteroides & $7327 \pm 2934.8$ & 14.32 & $2300 \pm 1020.4$ & 5.36 & 0.137 \\
Cyanobacteria & $286 \pm 88.3$ & 0.56 & $214 \pm 58.1$ & 0.50 & 0.508 \\
Euryarchaeota & $400 \pm 97.3$ & 0.78 & $387 \pm 194.2$ & 0.90 & 0.951 \\
Firmicutes & $30320 \pm 4856.3$ & 59.26 & $33848 \pm 2811.8$ & 78.88 & 0.544 \\
Unidentified & $3504 \pm 1402.1$ & 6.85 & $2366 \pm 260.9$ & 5.51 & 0.443 \\
Proteobacteria & $697 \pm 225.8$ & 1.36 & $521 \pm 58.5$ & 1.22 & 0.468 \\
Saccharibacteria & $683 \pm 224.3$ & 1.34 & $121 \pm 95.3$ & 0.28 & 0.044 \\
Synergistetes & $1287 \pm 680.6$ & 2.52 & - & - & 0.088 \\
Tenericutes & $39 \pm 18.3$ & 0.08 & $196 \pm 47.3$ & 0.46 & 0.012 \\
Verrucomicrobia & $6075 \pm 1334.6$ & 11.87 & $2642 \pm 1012.8$ & 6.16 & 0.068 \\
\hline
\end{tabular}

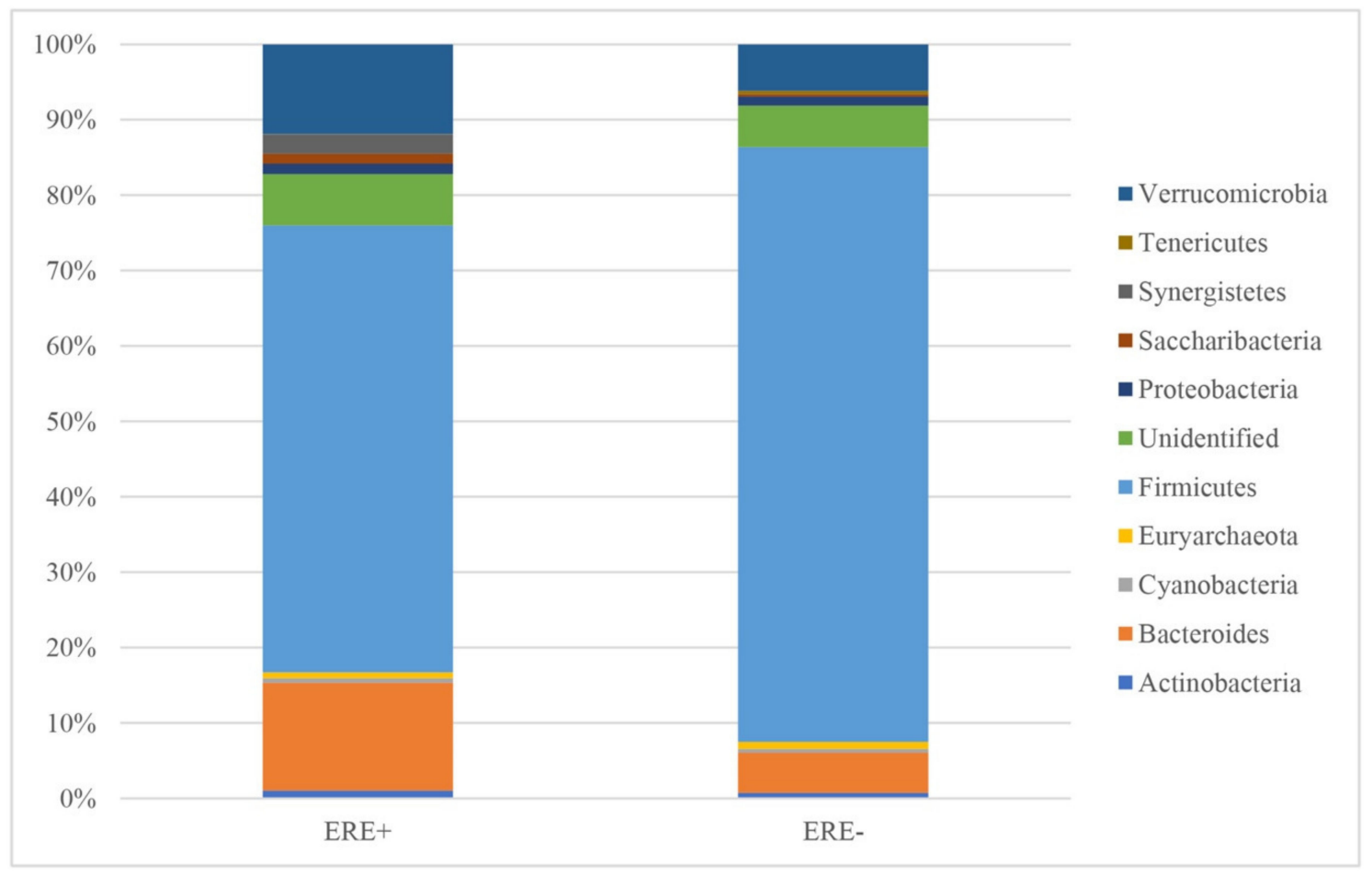

Figure 4. The relative abundance of bacterial genera in cecal samples of healthy and ERE rabbits. ERE+:

Rabbits positive for epizootic enteropathy. ERE-: Healthy rabbits.

Following statistical analysis, ERE-positive rabbits showed a significantly higher abundance for some identifiable microorganisms. These gave an indication of organisms that could be used as biomarkers. Specifically, these were: Akkermansia (15.22\% vs. $5.62 \%)$; Clostridium ( $3.56 \%$ vs. $0.61 \%)$; Bacteroides (2.67\% vs. $0.92 \%)$; Cloacibacillus (1.72\% vs. $0 \%$ ); Saccharimonas (1.27\% vs. $0.22 \%)$; Synergistes ( $0.71 \%$ vs. $0 \%)$; and Erysipelatoclostridium (0.49\% vs. $0.03 \%)$. In the case of archea, Methanosphaera $(0.58 \%$ vs. $0.09 \%)$ was the only genus that increased significantly. In addition, having the ERE disease significantly reduced the relative abundance of the genera Subdoligranulum (3.64\% vs. $7.49 \%)$ and Eisenbergiella ( $0.03 \%$ vs. $1.27 \%)$ and the families Ruminococcaceae $(26.34 \%$ vs. $34.12 \%)$ and Lachnospiraceae ( $8.14 \%$ vs. $23.49 \%$ ) (Figure 5). 


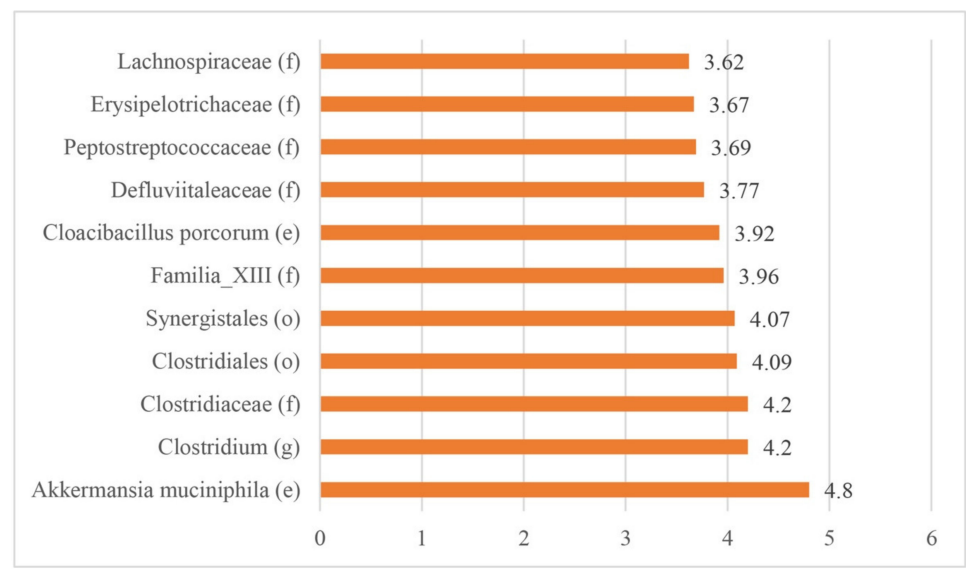

Figure 5. Biomarkers identified using the linear discriminant analysis effect size (LEfSe) test in rabbits with ERE. (f): family, (e): species, (o): order, (g): genus.

\subsubsection{Bacterial Diversity}

The diversity and richness of the microbiota of healthy rabbits was greater in all of the calculated indices compared to EEC-positive rabbits (Table 3).

Table 3. Summary of sequences identified and comparison of microbial diversity indices in rabbit cecal contents of animals with and without ERE. ERE+: Rabbits positive for epizootic enteropathy. ERE-: Healthy rabbits.

\begin{tabular}{cccc}
\hline Sequences and Index. & ERE+ & ERE- & $p$-Value \\
\hline Number of sequences & 115,534 & 99,863 & \\
Number of operational taxonomic units (OTU) & 317 & 279 & \\
Number of identified genera & 76 & 81 & \\
Shannon Index & $6.24(0.18)$ & $6.54(0.04)$ & 0.001 \\
Simpson Index & $0.95(0.04)$ & $0.97(0.01)$ & 0.001 \\
Simpson Inverse Index & $30.12(0.09)$ & $43.54(0.06)$ & 0.001 \\
Fisher Index & $66.20(2.19)$ & $81.40(0.80)$ & 0.001 \\
Chao1 Index & $304.98(6.70)$ & $275.86(2.50)$ & 0.002 \\
\hline
\end{tabular}

\subsubsection{Biomarkers}

The LEfSe test reported 11 biomarkers in animals with ERE disease; however, 6 biomarkers were reported in healthy rabbits (Figures 5 and 6).

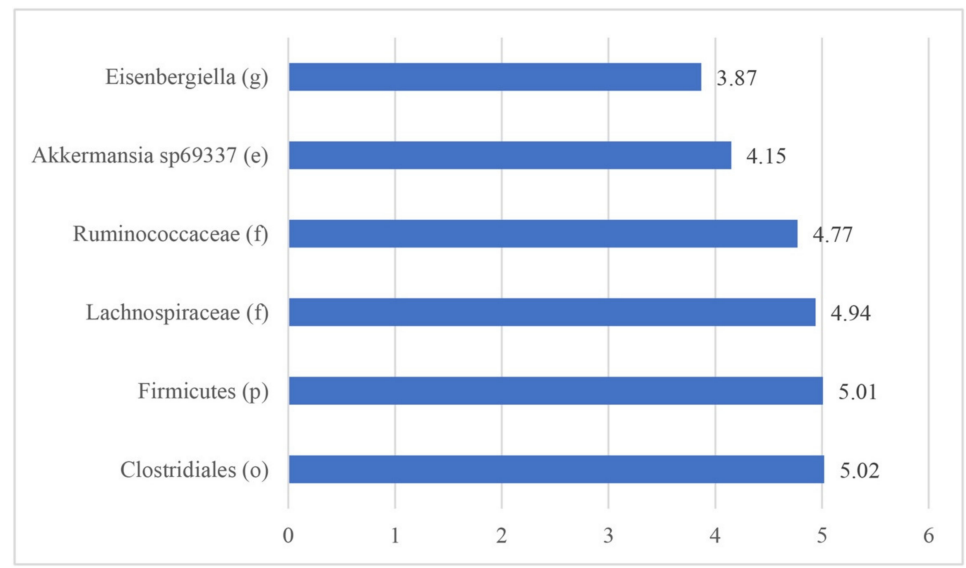

Figure 6. Biomarkers identified using the LEfSe test in healthy rabbits. (f): family, (e): species, (o): order, (g): genus, (p): phylum. 


\section{Discussion}

Macroscopic lesions observed were similar to those mentioned previously in several studies $[4,9,11,28]$, giving us confidence that those assumed to be ERE positive at the outset were indeed suffering from ERE. Interestingly, however, we observed atrophy of the intestinal crypts in this study. This could be associated with two aspects caused by the syndrome: (1) The absence of food in the colon due to impaction of the cecum, and (2) the proliferation of microbial communities belonging to the family Clostridiaceae. In the case of microbial proliferation leading to digestive problems, this has been documented in broiler chickens [29] and children [30]. Cecal impaction promotes an environment for the proliferation of various pathogenic communities, and also causes food to not access the more distal sections of the gastrointestinal tract and so decreases the growth of resident communities, such as Ruminococcus spp. [31].

The mean number of sequences obtained during this analysis was around 14,500 for ERE+ animals and around 12,500 for ERE- animals. These values are slightly lower than some examples of previous works involving rabbits, e.g., mean of around 37,500 per sample [32] and 48,700 per sample [33]. However, they are still higher than similar works published on other herbivore systems, e.g., around 5500 per cow [34] and around 10,000 per deer [35]. However, the rarefaction curve showed good coverage and the debugging carried out by the DADA2 algorithm reinforces the accuracy of the identified OTUs and reduces bioinformatical error [21]. Comparison of the metataxonomic profiles showed a difference between groups $(p<0.04)$, which supported the classification of this enteropathy as dysbiosis. It has been reported that ERE dysbiosis affects a rabbit's microbiota, because it increases the abundance of the phyla Bacteroidetes, Proteobacteria, and Verrucomicrobia [15,17]. However, it decreases the abundance of the phylum Firmicutes $[15,17,18]$. In addition, an effect on the diversity has been reported, principally on the genera Butyricimonas and Anaerotruncus [17].

In this study, an increase in abundance for the phyla Bacteroidetes, Saccharibacteria, Tenericutes, and Verrucomicrobia in ERE-infected animals was observed, but the phylum Firmicutes decreased. However, due to intra-group variation in the numbers, only Saccharibacteria and Tenericutes showed significant $(p<0.05)$ increases, with Verrucomicrobia showing a trend towards an increase $(p=0.068)$. Furthermore, the Synergistetes phylum was only found in ERE rabbits. However, the present study did not record the presence of Escherichia-Shigella or a significant change in the phylum Proteobacteria. Abecia et al. [17] already reported that the Synergistetes phylum has only been seen in rabbits with ERE; however, more recently it has been shown that it can also appear in healthy rabbits [18]. Furthermore, a recent study did not report the Synergistetes phylum in young rabbits [33]. In the case of Escherichia-Shigella, this genus has not been reported in some animals with ERE [17,33]; however, other studies showed that the genus can be present either in healthy or ERE rabbits [15,18].

The work presented here shows some differences in terms of the sequences detected relative to previous reports. Unlike many of the previous investigations into the microbiome of animals infected with ERE relative to healthy animals, we did not use a round of PCR prior to the sequencing stage, instead sequencing directly from the genomic DNA. This approach was used as potentially there have been differences between studies in the microbial profile, which may be related to the type of PCR amplification used prior to performing sequencing. For example, it has been shown that a prior round of PCR can present different forms of bias in terms of the sequences generated. The primers, which are described as being "universal", are not always as universal as first described and may fail to amplify all intended targets [36,37]. In addition, although PCR is regarded as a reliable process, it is not error free and so amplification errors can in turn lead to sequencing errors down the line [36,37]. For this reason, some papers have suggested that, if possible, it may be prudent to forego any steps prior to sequencing, including initial rounds of PCR [38]. Moreover, in the case of PCR being performed prior to sequencing, this has been shown to have a distorting effect when using second-generation platforms, including affecting the calculations of diversity and relative abundance [39-41]. For the reasons given above, we decided that it was appropriate to consider a pre-sequencing round of PCR as an optional requirement. In doing so, we feel that the current data avoids issues associated with PCR bias and so 
should provide a more accurate reflection of the genomic DNA available for analysis at the point of DNA extraction. Given the fact that we managed to identify sequences from organisms not previously reported in the digestive tract of the rabbit, we feel this decision has been vindicated.

Other factors that could contribute to the differences relative to previous work could include the experimental diet, which can contribute to the stability of the phylum Proteobacteria. In the present study, the diet did not contain any type of antibiotic, another factor that was different from the work of Abecia et al. [17], where the diet offered contained antibiotics (bacitracin and robenidine). Other dietary variables between experiments also include the level of crude fiber (CF) and crude protein $(\mathrm{CP})$, which are known to be important factors in terms of preventing, or reducing, the issue of ERE.

The increase in the abundance and decrease in diversity indices observed in this study corroborates that reported in some previous studies $[15,17]$. The Simpson index differs from that reported by Jin et al. [18], who did not detect a difference. This index does not discriminate between species with a low population and diversity is confirmed by observing other diversity indices, in which differences can be observed, as we showed in this study. In this study, the relative abundance of organisms in those with ERE was higher than in healthy animals, and the Chao1 index was 304.98. Previously, the ERE Chao1 index has been reported as 180.01 [17] and 191.4 [18]. However, the relative abundance in healthy rabbits was similar to those in earlier studies $[17,18]$. Differences in the values obtained in this study may be associated with the origin of the rabbits and the type of food offered; the rabbits were obtained from production farms and no type of antibiotic was offered in the diet unlike Abecia et al. [17], who had antibiotics in the diet, which may have an effect on the microbial profile [8,42]. In the case of Jin et al. [18], antibiotics were not offered, but rabbits were free of specific pathogens and were maintained under laboratory conditions, which could have a strong effect on the establishment of the colonization of microorganisms and can affect the diversity and abundance of the microbiota.

Members of the phyla Verrucomicrobia (family Verrucomicrobiaceae), Firmicutes (families Clostridiaceae, Familia_XIII, Defluviitaleaceae, Peptostreptococcaceae, Erysipelotrichaceae, Lachnospiraceae), and Synergistetes (family Synergistaceae) were associated with the presence of ERE by LEfSe analysis (Figure 5). In addition, the following organisms were also found to be associated with ERE: Akkermancia muciniphila, Clostridium spp., and Cloacibacillus porcorum.

The Firmicutes phylum is the most abundant within the cecal microbiota of healthy rabbits, representing between $65 \%$ to $75 \%$ of the abundance [43-45]. In the present study, Firmicutes were the most abundant organisms in both groups, although the relative abundance was higher in healthy rabbits at $78.88 \%$, relative to those with ERE with $59.26 \%$, in keeping with previous studies $[15,17,18]$.

We observed the Lachnospiraceae family in both healthy and ERE rabbits, in keeping with studies of Bäuerl et al. [15] and Abecia et al. [17], but unlike Jin et al. [18], who only found this family in healthy rabbits.

Clostridium spp. have been linked to ERE, primarily C. perfringens and C. cuniculli [7,31,46,47]. Djukovic et al. [31] identified some strains of $C$. cuniculli and proposed that in association with other Clostridium spp., they were involved in the development of ERE, although inoculation with them has not produced enteropathy. In this study, we found that Clostridium as a genus is associated with the presentation of ERE.

The phylum Verrucomicrobia, although present in healthy animals [43,44], has been implicated with the presence of ERE when it has been increased between $6 \%$ to $9.5 \%[15,18]$. In the present study, the phylum Verrucomicrobia had an increase of 9.6\% in ERE-infected animals and one species within the phylum was identified as an ERE biomarker: Akkermansia muciniphila.

Akkermansia muciniphila has been associated with ERE previously $[15,17,18]$, and it is known to degrade mucins and stimulate mucin production in the gastrointestinal tract [48]. Therefore, its presence can be related to the increase in mucus production in the small intestine and mucus accumulation in the cecum. Interestingly, human dysbiosis studies $[49,50]$ showed that a low presence of this bacterium is associated with the presentation of ulcerative colitis, irritable bowel syndrome, and obesity. Moreover, the role that this microorganism plays in the preservation of the physical barrier of the intestine has 
been verified by stimulating the production of mucins [51-53]. However, the proliferation of this bacterium in ERE syndrome may be related either with the stimulation of globet cells or the interaction with other bacteria due to the disbalance of the bacterial population.

The Synesrgistetes phylum is a common inhabitant of the soil, as well as in anaerobic systems, such as the gastro-intestinal tract of pigs $[19,54]$. It has been associated with being an opportunistic pathogen in pigs [19] and an agent associated with oral dysbiosis in cattle [20]. Normally, they degrade mucin and show resistance to vancomycin [20]. Cloacibacillus porcorum belongs to the phylum Synergistetes and it can ferment amino acids and uses mucus as a carbon source [55]. In the present study, C. porcorum was identified only in ERE rabbits. Jin et al. [18] also reported C. porcorum in ERE rabbits but found it at a lower percentage in healthy rabbits. Some studies did not find any members of this phylum $[5,17]$, while others $[33,43,44]$ failed to identify this phylum in the cecal microbiota of healthy rabbits, and others [56] reported it in the hard feces of healthy rabbits. The presence of the genus may be associated with mucus production.

Metataxonomic studies can generate information that allows the theorizing and testing of specific treatment ideas for ERE. In this study, some bacteria were identified as potential ERE biomarkers, which appear to be strongly related with the disease. Recently, Read et al. [33] showed that cecal microbiota development starts from an early age under different feeding conditions (lactation and solid food), and the length of lactation time has a directly proportional effect with the cecal health of rabbits. Potentially poor handling during lactation and nursing can have a high impact on the cecal microbiota and can contribute to ERE syndrome development. Therefore, it is necessary to undertake some interaction experiments and more metataxonomic studies to elucidate the etiopathology of this syndrome and subsequently propose alternative treatments. The data presented here provides a new insight into candidate organisms that may be used as biomarkers to identify animals that may be susceptible to the development of ERE.

\section{Conclusions}

Rabbit epizootic enteropathy is a dysbiosis that presents a principally smaller size of the crypts in the colon. Microbial imbalance damages are related with the proliferation of communities belonging to the phyla Verrucomicrobia; (Akkermansia muciniphila), Firmicutes (Clostridium spp.), and Synergestes (e.g., Cloacibacillus porcorum). This is the first time that Cloacibacillus porcorum has been reported to have an association with ERE disease.

Supplementary Materials: The following are available online at http://www.mdpi.com/2076-2615/10/6/936/s1, Table S1; title: Relative abundance of the Phyla, Classes, Orders, Families and Genera in the caecal and faecal samples. Values with an abundance value of less than 0.001 are denoted as "low".

Author Contributions: Conceptualization A.M.O.-R.; methodology, X.-H.D.P.-P., J.G.G.-S., bioinformatic and statistical analysis, X.-H.D.P.-P., R.C.A.-M. resources, A.M.O.-R., J.G.G.-S.; writing-original draft preparation X.-H.D.P.-P.; writing-review and editing, A.M.O.-R., N.R.M. All authors have read and agreed to the published version of the manuscript.

Funding: This research was funded by University grant number (Foper-2018-00212).

Acknowledgments: With thanks to Laura Escobar-Salazar, who loaned installations to do the experiment.

Conflicts of Interest: The funders had no role in the design of the study; in the collection, analyses, or interpretation of data; in the writing of the manuscript, or in the decision to publish the results. The authors declare no conflict of interest.

\section{References}

1. Licois, D.; Vautherot, J.F.; Coudert, P.; Dambrine, G. Modele de Reproduction Experimentale de L'enterocolite Epizootique Chez Des Lapins Eops. World Rabbit Sci. 1998, 6, 349-353. [CrossRef]

2. Bouquin, S.; Jobert, J.L.; Larour, G.; Balaine, L.; Eono, F.; Boucher, S.; Huneau, A.; Michel, V. Risk Factors for an Acute Expression of Epizootic Rabbit Enteropathy Syndrome in Rabbits after Weaning in French Kindling-to-Finish Farms. Livest. Sci. 2009, 125, 283-290. [CrossRef] 
3. Lebas, F.; Coudert, P.; de Rochambeau, H.; Thebault, R.G. El Conejo Cría y Patología. Collecion FAO: Producción y Sanidad Animal; FAO: Rome, Italy, 1996; Volume 19, ISSN 1014-6423.

4. De Rozas Ruiz de Gauna, A.M.P.; Carabaño, R.; García, J.; Rosell, J.M.; Díaz Cano, J.V.; Barbé Garcia, J.; Pascual Amorós, J.J.; Badiola Saíz, J.I. Etiopatogenia de la enteropatía epizoótica del conejo. Boletín de Cunicultura 2005, 139, 167-174.

5. Rodríguez Serrano, T.M. La ERE, enteropatía epizoótica del conejo. Boletín de Cunicultura 2004, 135, 39-46.

6. Pérez, C.; Pérez, A.; Coscelli, A.; Prieto, A.; Díaz-Baños, P. Identificación de infecciones digestivas en conejos: Orientaciones para optimizar la prevención y el control. Boletín de cunicultura 2015, 176, 14-18.

7. Pérez, J.E. Revisión y conclusiones de la enteropatía epizoótica del conejo. Cunicultura 2013, 38, 9-12.

8. Abecia, L.; Fondevila, M.; Balcells, J.; McEwan, N.R. The effect of lactating rabbit does on the development of the caecal microbial community in the pups they nurture. J. Appl. Microbiol. 2007, 103, 557-564. [CrossRef]

9. Licois, D.; Wyers, M.; Coudert, P. Epizootic Rabbit Enteropathy: Experimental transmission and clinical characterization. Vet. Res. 2005, 36, 601-613. [CrossRef]

10. Dewrée, R.; Meulemans, L.; Lassence, C.; Desmecht, D.; Ducatelle, R.; Mast, J.; Licois, D.; Vindevogel, H.; Marlier, D. Experimentally induced epizootic rabbit enteropathy: Clinical, histopathological, ultrastructural, bacteriological and haematological findings. World Rabbit Sci. 2007, 15, 91-102. [CrossRef]

11. Licois, D.; Coudert, P.; Cerén, N.; Vautherot, J.-F. Epizootic Enterocolitis of the rabbit: Review of current research. World Rabbit Sci. 2000, 8 (Suppl. 1), 187-194.

12. Fernández, G. Enfermedades Infecciosas que cursan con procesos digestivos en conejos. Boletín de Cunicultura Lagomorpha 2006, 144, 23-40.

13. Haligur, M.; Ozmen, O.; Demir, N. Pathological and Ultrastructural Studies on Mucoid Enteropathy in New Zealand Rabbits. J. Exot. Pet Med. 2009, 18, 224-228. [CrossRef]

14. Allen, A.L.; Bryant, U.K. What's Up Doc? Can. Vet. J. 2009, 50, 1297-1299. [PubMed]

15. Bäuerl, C.; Collado, M.C.; Zúñiga, M.; Blas, E.; Pérez Martínez, G. Changes in Cecal Microbiota and Mucosal Gene Expression Revealed New Aspects of Epizootic Rabbit Enteropathy. PLoS ONE 2014, 9, e105707. [CrossRef] [PubMed]

16. Huybens, N.; Horueix, J.; Licois, D.; Mainil, J.; Marlier, D. Pyrosequencing of epizootic rabbit enteropathy inocula and rabbit caecal samples. Vet. J. 2013, 196, 109-110. [CrossRef]

17. Abecia, L.; Rodríguez-Romero, N.; Martínez-Fernández, G.; Martínez-Vallespín, B.; Fondevila, M. Pyrosequencing study of cecal bacterial community of rabbit does and kits from a farm affected by epizootic rabbit enteropathy. World Rabbit Sci. 2017, 25, 261-272. [CrossRef]

18. Jin, D.X.; Zou, H.W.; Liu, S.Q.; Wang, L.Z.; Xue, B.; Wu, D.; Tian, G.; Cai, J.; Yan, T.H.; Wang, Z.S.; et al. The underlying microbial mechanism of epizootic rabbit enteropathy triggered by a low fiber diet. Sci. Rep. 2018, 8, 12489. [CrossRef]

19. Park, S.-J.; Kim, J.; Lee, J.-S.; Rhee, S.-K.; Kim, H. Characterization of the fecal microbiome in different swine groups by high-throughput sequencing. Anaerobe 2014, 28, 157-162. [CrossRef]

20. Borsanelli, A.C.; Lappin, D.F.; Viora, L.; Bennett, D.; Dutra, I.S.; Brandt, B.W.; Riggio, M.P. Microbiomes associated with bovine periodontitis and oral health. Vet. Microbiol. 2018, 218, 1-6. [CrossRef]

21. Callahan, B.J.; McMurdie, P.J.; Rosen, M.J.; Han, A.W.; Johnson, A.J.A.; Holmes, S.P. DADA2: High-resolution simple inference from Illumina amplicon data. Nat. Methods 2016, 13, 581-583. [CrossRef]

22. Norma Oficial Mexicana NOM-062-ZOO-1999. Especificaciones técnicas para la producción, cuidado y uso de los animales de laboratorio. Sección 9. Available online: http://www.fmvz.unam.mx/fmvz/principal/arch ivos/062ZOO.PDF (accessed on 25 January 2019).

23. Gómez Soto, J.G.; Reis de Souza, T.C.; Mariscal Landin, G.; Aguilera Barreyro, A.; Bernal Santos, M.G.; Escobar Garcia, K. Gastrointestinal morphophysiology and presence of kafirins in ileal digesta in growing pigs fed sorghum-based diets. J. Appl. Anim. Res. 2018, 46, 618-625. [CrossRef]

24. Steel, R.G.D.; Torrie, J.H. Principles and Procedures of Statistics: A Biometrical Approach, 2nd ed.; McGraw-Hill: New York, NY, USA, 1980.

25. Bolger, A.M.; Lohse, M.; Usadel, B. Trimmomatic: A flexible trimmer for Illumina sequence data. Bioinformatics 2014, 30, 2114-2120. [CrossRef] [PubMed]

26. Oksanen, J.; Blanchet, F.G.; Friendly, M.; Kindt, R.; Legendre, P.; McGlinn, D.; Minchin, P.R.; O’Hara, R.B.; Simpson, G.L.; Solymos, P.; et al. Community Ecology Package. 2019. Available online: https://github.com/v egandevs/vegan (accessed on 23 June 2019). 
27. The R Foundation. Introduction to R. Disponible, 2019. Available online: https://www.r-project.org/about.ht $\mathrm{ml}$ (accessed on 20 May 2020).

28. Rodríguez-De Lara, R.; Cedillo-Peláez, C.; Constantino, F.; Fallas-López, M.; Cobos-Peralta, M.A.; Gutiérrez-Olvera, C.; Juárez-Acevedo, M.; Miranda-Romero, L.A. Studies on the evolution, pathology, and immunity of commercial fattering rabbits affected with epizootic outbreaks of diarrhoeas in México: A case report. Research in Veterinary Sci. 2008, 84, 257-268. [CrossRef] [PubMed]

29. Aslam, S.; Durrani, A.Z.; Rizwan, M.; Kashif, M. Comparative Efficacy of metronidazole in combination colistinsulphate and probiotics against Clostridium perfringens and their effects on histopathological features of visceral organs in broiler. Veterinaria 2016, 4, 11-17.

30. Butler, D.; Torres-Torres, S.; Pahud, B.; Myers, A.; Bass, J.A.; St Peter, S.D.; McCulloh, R. Clostridium difficile ileitis in pediatric inflammatory bowel disease. J. Pediatric Gastroenterol. Nutr. 2017, 64, e156-e158. [CrossRef]

31. Djukivic, A.; García-Garcera, M.; Martínez-Paredes, E.; Issac, S.; Artacho, A.; Martínez, J.; Ubeda, C. Gut colonization by a novel Clostridium species is associated with the onset of epizootic rabbit enteropathiy. Vet. Res. 2018, 49, 213. [CrossRef]

32. Crowley, E.J.; Kink, J.M.; Wilkinson, T.; Worgan, H.J.; Huson, K.M.; Rose, M.T.; McEwan, N.R. Comparison of the microbial population in rabbits and guinea pigs by next generation sequencing. PLoS ONE 2017, 12, e0165779. [CrossRef]

33. Read, T.; Fortun-Lamothe, L.; Pascal, G.; Le Boulch, M.; Cauquil, L.; Gabinaud, B.; Banneller, C.; Balmisse, E.; Destombes, N.; Bouchez, O.; et al. Diversity and co-occurrence pattern analysis of cecal microbiota establishment at the onset of solid feeding in young rabbits. Front. Microbiol. 2019, 10, 973. [CrossRef]

34. Fouts, D.E.; Szpakowski, S.; Purushe, J.; Torralba, M.; Waterman, R.C.; MacNeil, M.D.; Alexander, L.J.; Nelson, K.E. Next Generation Sequencing to Define Prokaryotic and Fungal Diversity in the Bovine Rumen. PLOS ONE 2012, 7, e48289. [CrossRef]

35. Gruninger, R.J.; Sensen, C.W.; McAllister, T.A.; Forster, R.J. Diversity of Rumen Bacteria in Canadian Cervids. PLoS ONE 2014, 9, e89682. [CrossRef]

36. Zhou, X.; Li, Y.; Liu, S.; Yang, Q.; Su, X.; Zhou, L.; Tang, M.; Fu, R.; Li, J.; Huang, Q. Ultra-deep sequencing enables high-fidelity recovery of biodiversity for bulk arthropod samples without PCR amplification. Gigascience 2013, 2, 4. [CrossRef] [PubMed]

37. Zaura, E. Next-generation Sequencing Approaches to Understanding the Oral Microbiome. Adv. Dent. Res. 2016, 24, 81-85. [CrossRef] [PubMed]

38. Ebbert, M.T.W.; Wadsworth, M.E.; Staley, L.A.; Hoyt, K.L.; Pickett, B.; Miller, J.; Duce, J.; Kauwe, J.S.K.; Ridge, P.G. Evaluating the necessity of PCR duplicate removal from next-generation sequencing data and a comparison of approaches. BMC Bioinform. 2016, 17, 239. [CrossRef] [PubMed]

39. Aird, D.; Ross, M.G.; Chen, W.-S.; Danielsson, M.; Fennell, T.; Russ, C.; Jaffe, D.B.; Nusbaum, C.; Gnirke, A. Analyszing and minimizing PCR amplification bias in Illumina sequencing libraries. Genome Biol. 2011, 12, 18. [CrossRef] [PubMed]

40. Kebschull, J.M.; Zador, A.M. Sources of PCR-induced distortions in high-throughput sequencing data sets. Nucleic Acids Res. 2015, 43, e143. [CrossRef]

41. Tilak, M.K.; Botero-Castro, F.; Galtier, N.; Nabholz, B. Illumina library preparation for sequencing the GC-rich frection of heterogeneous genomic DNA. Genome Biol. Evol. 2018, 10, 616-622. [CrossRef]

42. Boisot, P.; Duperray, J.; Guyonvarch, A.; Richard, A.; Licois, D.; Coudert, P. Evaluation of the effectiveness of soluble bacitracin (Bacivet $s^{\circledR}$ ) in drinking water compared to bacitracin in the feed (Albac $\left.{ }^{\circledR}\right)$, during an experimental reproduction of epizootic rabbit enteropathy syndrome. México. In Proceedings of the 8th World Rabbit Congress, Puebla, Mexico, 7-10 September 2004; pp. 457-462.

43. Abecia, L.; Fondevila, M.; Balcells, J.; Edwards, J.E.; Newbold, C.J.; McEwan, N.R. Molecular profiling of bacterial species in the rabbit caecum. FEMS Microbiol. Lett. 2005, 244, 111-115. [CrossRef]

44. Mattioli, S.; Dal Bosco, A.; Combes, S.; Moscati, L.; Crotti, S.; Cartoni, A.; Cotozzolo, E.; Castellini, C. Dehydrated alfalfa and fresh grass supply in young rabbits: Effect on performance and cecal microbiota biodiversity. Animals 2019, 9, 341. [CrossRef]

45. Yuan, M.; Liu, S.; Wang, Z.; Wang, L.; Xue, B.; Zou, H.; Tian, G.; Cai, J.; Peng, Q. Effects of particle size of ground alfalfa on cecal bacteria and archaea populations of rabbits. Peer] 2019, 7, e7910. [CrossRef] 
46. De Blas, J.C.; Astillero, J.R.; Chamorro, S.; Corujo, A.; García-Alonso, J.; García-Rebollar, P.; García-Ruiz, A.I.; Menoyo, D.; Nicodemus, N.; Romero, C.; et al. Efectos de la nutrición y el manejo sobre el desarrollo de patologías digestivas de gazapos en un entorno de enteropatía epizoótica. 2007. Salud intestinal en gazapos. FEDNA. pp. 109-124. Available online: http://fundacionfedna.org/sites/default/files/07CAP_VI.pdf (accessed on 26 March 2020).

47. Chamorro, S.; De Blas, C.; Grant, G.; Badiola, I.; Menoyo, D.; Carabaño, R. Effect of dietary supplementation of glutamine-arginine on intestinal health in twenty-five-day-old weaned rabbits. J. Anim. Sci. 2010, 88, 170-180. [CrossRef]

48. Macchione, I.G.; Lopetuso, L.R.; Ianiro, G.; Napoli, M.; Gibiino, G.; Rizzatti, G.; Petito, V.; Gasbarrini, A.; Scaldaferri, F. Akkermansia muciniphila: Key player in metabolic and gastrointestinal disorders. Eur. Rev. Med. Pharmacol. Sci. 2019, 23, 8075-8083. [CrossRef] [PubMed]

49. López-Siles, M.; Enrich-Capó, N.; Aldeguer, X.; Sabat-Mir, M.; Duncan, S.H.; García-Gil, L.J.; Martínez-Medina, M. Alterations in the Abundance and Co-occurrence of Akkermansia muciniphila and Faecalibacterium prausnitzii in the Colonic Mucosa of Inflammatory Bowel Disease Subjects. Front. Cell. Infect. Microbiol. 2018, 8, 281. [CrossRef] [PubMed]

50. Earley, H.; Lennon, G.; Balfe, A.; Coffey, J.C.; Winter, D.C.; O’Connell, P.R. The abundance of Akkermansia muciniphila and its relationship with sulphated colonic mucins in health and ulcerative colitis. Sci. Rep. Nat. Re. 2019, 9, 15683. [CrossRef] [PubMed]

51. Derrien, M.; Belzer, C.; M de Vos, W.M. Akkermansia muciniphila and its role in regulating host functions. Microb. Pathog. 2017, 106, 171-181. [CrossRef]

52. Plovier, H.; Everard, A.; Druart, C.; Depommier, C.; Van Hul, M.; Geurts, L.; Chilloux, J.; Ottman, N.; Duparc, T.; Lichtenstein, L.; et al. A purified membrane protein from Akkermansia miciniphila or the pasteurized bacterium improves metabolism in obese and diabetic mice. Nat. Med. 2016, 23, 107-113. [CrossRef]

53. Bian, X.; Wu, W.; Yang, L.; Lv, L.; Wang, Q.; Li, Y.; Ye, J.; Fang, D.; Wu, J.; Jiang, X.; et al. Administration of Akkermansia muciniphila Ameliorates Dextran Sulfate Sodium-Induced Ulcerative Colitis in Mice. Front. Microbiol. 2019, 10, 2259. [CrossRef]

54. Vo, N.; Tsai, T.C.; Maxwell, C.; Carbonero, F. Early exposure to agricultural soil accelerates the maturation of the early-life pig gut microbiota. Anaerobe 2017, 45, 31-39. [CrossRef]

55. Looft, T.; Levine, U.Y.; Stanton, T.B. Cloacibacillus porcorum sp. nov., a mucin-degrading bacterium from the swine intestinal tract and emended description of the genus Cloacibacillus. Int. J. Syst. Evol. Microbiol. 2013, 63, 1960-1996. [CrossRef]

56. Eshar, D.; Weese, J.S. Molecular analysis of the microbiota in hard feces from healthy rabbits (Oryctolagus cuniculus) medicated with long term oral meloxicam. BMC Vet. Res. 2014, 10, 62. [CrossRef] 